\title{
WEAK-INVARIANT PROPERTIES OF THE NORM TOPOLOGY
}

\author{
I. NAMIOKA AND R. POL
}

(Communicated by William J. Davis)

\begin{abstract}
A property $(\mathrm{P})$ relative to the norm topology of a Banach space is a weak-invariant if, whenever $A$ and $B$ are weakly homeomorphic subsets of (possibly different) Banach spaces and $(A$, norm) has property $(\mathrm{P})$, then $(B$, norm $)$ has property $(\mathrm{P})$. We show that the property of being $\sigma$-discrete and the property of being an absolute Souslin- $\mathscr{F}$ space of weight $\leq \aleph_{1}$, both relative to the norm topology, are weak-invariants. These conclusions are obtained from a result concerning maps of metrizable spaces into function spaces.
\end{abstract}

It is a well-known fact that a weakly separable subset of a Banach space is separable relative to the norm topology. One can formalize this observation as follows: a property $(\mathrm{P})$ relative to the norm topology is called a weak-invariant if, whenever $A$ and $B$ are subsets of (possibly different) Banach spaces such that $(A$, weak) and $(B$, weak) are homeomorphic and $(A$, norm) has property $(\mathrm{P})$, then $(B$, norm $)$ also has property $(\mathrm{P})$. Thus the separability relative to the norm topology and, indeed, the weight relative to the norm topology are weakinvariants.

One of the consequences of the theorem in this note is that the $\sigma$-discreteness relative to the norm topology is a weak-invariant. By combining this result and those in [5, 2], we also show that the property of being an absolute Souslin-F $\mathscr{F}$ space (defined later) of weight $\leq \aleph_{1}$ in the norm topology is also a weakinvariant. A subset $A$ of a metrizable space $M$ is said to be $\sigma$-discrete if $A=$ $\bigcup_{n=1}^{\infty} A_{n}$ with each $A_{n}$ relatively discrete. If $\rho$ is a metric on $M$ compatible with the topology, then the set $A$ is $\sigma$-discrete if and only if $A=\bigcup_{n=1}^{\infty} B_{n}$, where, for each $n$, there exists an $\varepsilon_{n}>0$ such that $\rho(x, y) \geq \varepsilon_{n}$ whenever $x, y \in B_{n}$ and $x \neq y$ (cf. [6]). We use repeatedly the fact that a subset $A$ of a metrizable space $M$ is $\sigma$-discrete if it is locally $\sigma$-discrete, i.e., for each $x \in M$ there is a neighborhood $U$ of $x$ such that $U \cap A$ is $\sigma$-discrete [6, Lemma 2]. In this note, all topological spaces are assumed to be Hausdorff.

Let $K$ be a compact space. Then $C(K)$ denotes the Banach space of all realvalued continuous functions on $K$ with the supremum norm. The topology of pointwise convergence is denoted by $\tau_{p}$. The following is the main result of the note.

Received by the editors October 3, 1991.

1991 Mathematics Subject Classification. Primary 46B26, 54C35; Secondary 54H05.

Key words and phrases. Weak topology of Banach spaces, $\sigma$-discreteness, Souslin sets. 
Theorem. Let $K$ be a compact space, let $M$ be a metrizable space, and let $u: M \rightarrow\left(C(K), \tau_{p}\right)$ be a continuous one-to-one map. If $u(M)$ is $\sigma$-discrete relative to the norm topology, then $M$ is $\sigma$-discrete.

As preparation for the proof of the theorem, we list three lemmas. The first one is an immediate consequence of Stone's well-known "lattice formulation" of the Stone-Weierstrass theorem [7; 4, p. 241].

Lemma 1. Let $K$ be a compact space and let $L$ be a subset of $C(K)$ which is a lattice, i.e., $\max (f, g)$ and $\min (f, g)$ are in $L$ whenever $f, g \in L$. Then the closure of $L$ in $C(K)$ relative to $\tau_{p}$ coincides with the norm closure of $L$.

The next two lemmas are concerned with the $\sigma$-discreteness. The proof of Lemma 3 can be given by repeating an argument in [5], but we give it here for the convenience of the readers.

Lemma 2. Let $\mathscr{M}=\left\{M_{t}: t \in T\right\}$ be a pairwise disjoint collection of $\sigma$-discrete subsets of a metrizable space $M$ such that each selector $S$ for $\mathscr{M}$ (i.e., $S \cap M_{t}$ is a singleton for each $t \in T)$ is $\sigma$-discrete. Then the union $\bigcup \mathscr{M}$ is $\sigma$-discrete.

Proof. Let $\rho$ be a compatible metric on $M$. Then for each $t \in T, M_{t}=$ $\bigcup_{n=1}^{\infty} M_{t}^{n}$ where $\rho(x, y) \geq \varepsilon(t, n)>0$ for each pair of distinct points $x, y$ in $M_{t}^{n}$. For $(k, n) \in \mathbb{N} \times \mathbb{N}$, let

$$
M_{k n}=\bigcup\left\{M_{t}^{n}: \varepsilon(t, n)>1 / k\right\} .
$$

If $B$ is a $(1 / 2 k)$ ball in $M$, then $B \cap M_{k n}$ is contained in a selector for $\mathscr{M}$ since $B \cap M_{t}^{n}$ is at most a singleton whenever $\varepsilon(t, n)>1 / k$. Hence by hypothesis, $B \cap M_{k n}$ is $\sigma$-discrete and so $M_{k n}$ is locally $\sigma$-discrete for each $(k, n)$. It follows from an earlier remark that each $M_{k n}$ and hence the countable union $\bigcup\left\{M_{k n}:(k, n) \in \mathbb{N} \times \mathbb{N}\right\}=\bigcup \mathscr{M}$ are $\sigma$-discrete.

The following notation will be used in the proof of the theorem as well as in the next lemma. Well-order the given metrizable space $M$ as $M=\left\{x_{\alpha}: \alpha<\mu\right\}$ where $\mu$ is the least ordinal with cardinality $|\mu|=|M|$ and, for each $\xi<\mu$, let

$$
M_{\xi}=\overline{\left\{x_{\alpha}: \alpha<\xi\right\}} .
$$

Also let

$$
\Gamma=\left\{\xi<\mu: \xi \text { is a limit ordinal and } M_{\xi} \backslash \bigcup\left\{M_{\alpha}: \alpha<\xi\right\} \neq \varnothing\right\} .
$$

Lemma 3. Suppose that $|\mu|$ is a regular cardinal and $M_{\xi}$ is $\sigma$-discrete for each $\xi<\mu$. If there is a function $\varphi: \Gamma \rightarrow \mu(=\{\alpha: \alpha<\mu\})$ such that $\varphi(\xi)<\xi$ for each $\xi \in \Gamma$ and $\left|\varphi^{-1}(\alpha)\right|<|\mu|$ for each $\alpha<\mu$, then $M$ is $\sigma$-discrete.

Proof. Extend the function $\varphi$ to $\varphi: \mu \rightarrow \mu$ by letting $\varphi(\xi+1)=\xi$ for $\xi<\mu$ and $\varphi(\xi)=\xi$ for each limit ordinal $\xi \in \mu \backslash \Gamma$. Then $\left|\varphi^{-1}(\alpha)\right|<|\mu|$ still holds for each $\alpha<\mu$. Let $\rho$ be a compatible metric for $M$, and let $B(A, \varepsilon)=\{x \in$ $M: \rho$-dist $(x, A)<\varepsilon\}$ for $A \subset M$ and $\varepsilon>0$. For $\xi<\mu$ and $n \in \mathbb{N}$, let

$$
M_{\xi}^{n}=M_{\xi} \backslash B\left(M_{\varphi(\xi)}, 1 / n\right) \quad \text { and } \quad M_{n}=\bigcup\left\{M_{\xi}^{n}: \xi<\mu\right\} .
$$

We show that $M_{n}$ is $\sigma$-discrete by showing that $M_{n}$ is locally $\sigma$-discrete. Let $x \in M$. Then $x \in M_{\xi_{0}}$ for some $\xi_{0}<\mu$. Since $\mu$ is regular and $\left|\varphi^{-1}(\alpha)\right|<|\mu|$ 
for each $\alpha<\mu$, there is a $\xi_{1}<\mu$ such that $\xi_{0}<\varphi(\xi)$ whenever $\xi_{1}<\xi<$ $\mu$. Then $\xi_{1}<\xi<\mu$ implies that $B\left(x, \frac{1}{n}\right) \subset B\left(M_{\varphi(\xi)}, \frac{1}{n}\right)$ and consequently $B\left(x, \frac{1}{n}\right) \cap M_{n} \subset M_{\xi_{1}}$ which is $\sigma$-discrete. To complete the proof, note that $\bigcup_{n=1}^{\infty} M_{n}=\bigcup\left\{M_{\xi} \backslash M_{\varphi(\xi)}: \xi<\lambda\right\}$, each $M_{\varphi(\xi)}$ being closed. Let $x \in M$ and let $\xi_{0}$ be the least ordinal with $x \in M_{\xi_{0}}$. If $\xi_{0}$ is a successor, then $\varphi\left(\xi_{0}\right)<\xi_{0}$. If $\xi_{0}$ is a limit ordinal, then $x \in M_{\xi_{0}} \backslash \bigcup\left\{M_{\alpha}: \alpha<\xi_{0}\right\}$. Hence $\xi_{0} \in \Gamma$ and $\varphi\left(\xi_{0}\right)<\xi_{0}$ by hypothesis. In either case $x \in M_{\xi_{0}} \backslash M_{\varphi\left(\xi_{0}\right)} \subset \bigcup_{n=1}^{\infty} M_{n}$. This shows that $M=\bigcup_{n=1}^{\infty} M_{n}$, and therefore $M$ is $\sigma$-discrete.

Proof of the Theorem. By dividing $M$ into a countable number of subsets, we may assume that for some $a>0$

$$
\|u(x)-u(y)\| \geq a \quad \text { for distinct } x, y \in M .
$$

Then, for each $A \subset M$,

$$
|\bar{A}|=|A| .
$$

To see this, let $L$ be the lattice generated by $u(A)$. If $A$ is finite then (4) is trivial. Otherwise, $|L|=|u(A)|=|A|, u$ being one-to-one. Let $\bar{L}$ be the norm-closure of $L$. Then by Lemma $1, \bar{L}$ is closed relative to $\tau_{p}$. Hence by the continuity of $u, u(\bar{A}) \subset \bar{L}$. Taking (3) into account, we see that $|\bar{A}|=$ $|u(\bar{A})| \leq|\bar{L} \cap u(M)| \leq|L|=|A|$.

We shall prove the theorem by induction on the cardinality of $M$. The conclusion of the theorem is trivially true if $M$ is countable. So assume that $|M| \geq \aleph_{1}$ and that the theorem is true for each metric space of cardinality less than $|M|$.

Case I. Assume that $|M|$ is singular. Then there exists a family $\mathscr{M}=\left\{M_{t}\right.$ : $t \in T\}$ of pairwise disjoint subsets of $M$ such that $M=\bigcup \mathscr{M},|T|<|M|$, and $\left|M_{t}\right|<|M|$ for each $t \in T$. By the inductive hypothesis, $M_{t}$ is $\sigma$-discrete for each $t \in T$, and each selector of $\mathscr{M}$, being of cardinality $|T|$, is also $\sigma$-discrete. Therefore $M$ is $\sigma$-discrete by Lemma 2 .

Case II. Assume that $|M|$ is regular, and recall the notation introduced just before Lemma 3. If $\xi<\mu$ is a limit ordinal, then

$$
M_{\xi}=\overline{\bigcup\left\{M_{\alpha}: \alpha<\xi\right\}}
$$

by (1). Furthermore, by (1) and (4), $\left|M_{\xi}\right|<|\mu|$ for each $\xi<\mu$. Hence by the inductive hypothesis, $\boldsymbol{M}_{\xi}$ is $\sigma$-discrete for each $\xi<\mu$.

By Lemma 3, the proof is complete if we can construct a function $\varphi: \Gamma \rightarrow \mu$ satisfying the conditions in the lemma. To this end, for each $\alpha<\mu$, let $L_{\alpha}$ be the lattice generated by $u\left(M_{\alpha}\right)$. Then

$$
\left|L_{\alpha}\right| \leq \max \left(\aleph_{0},\left|M_{\alpha}\right|\right)<|\mu| \quad \text { for each } \alpha<\mu .
$$

For $\xi \in \Gamma$, choose $x_{\xi} \in M_{\xi} \backslash \bigcup\left\{M_{\alpha}: \alpha<\xi\right\}$ (cf. (2)). By (5), $u\left(M_{\xi}\right)$ is contained in the $\tau_{p}$-closure of $\bigcup\left\{u\left(M_{\alpha}\right): \alpha<\xi\right\} \subset \bigcup\left\{L_{\alpha}: \alpha<\xi\right\}$ and $\bigcup\left\{L_{\alpha}: \alpha<\xi\right\}$ is a lattice. Therefore by Lemma $1, u\left(x_{\xi}\right)$ is in the normclosure of $\bigcup\left\{L_{\alpha}: \alpha<\xi\right\}$. Hence there are $\varphi(\xi)<\xi$ and $f_{\xi} \in L_{\varphi(\xi)}$ such that $\left\|u\left(x_{\xi}\right)-f_{\xi}\right\|<a / 2$. If $\xi$ and $\eta$ are distinct ordinals in $\Gamma$, then $x_{\xi} \neq x_{\eta}$ and so $\left\|u\left(x_{\xi}\right)-u\left(x_{\eta}\right)\right\| \geq a$ by (3). Hence $f_{\xi} \neq f_{\eta}$ or $\xi \rightarrow f_{\xi}$ is one-to-one. Hence by (6), $\left|\varphi^{-1}(\alpha)\right| \leq\left|\left\{\xi \in \Gamma: f_{\xi} \in L_{\alpha}\right\}\right| \leq\left|L_{\alpha}\right|<|\mu|$ for each $\alpha<\mu$. This completes the proof. 
If $E$ is a Banach space then $E$ can be considered to be a closed linear subspace of $C(K)$, where $K$ is the unit ball of the dual $E^{*}$ provided with the weak* topology. The topology of pointwise convergence on $K$ induces the weak topology on $E$. Hence the following corollary is the direct consequence of the theorem.

Corollary 1. Let $M$ be a metrizable space and $E$ a Banach space. If $u: M \rightarrow$ ( $E$, weak) is a continuous and one-to-one map and if $u(M)$ is $\sigma$-discrete relative to the norm topology, then $M$ is $\sigma$-discrete.

The following corollary is mentioned in the introduction.

Corollary 2. The $\sigma$-discreteness relative to the norm topology is a weak-invariant.

Proof. Let $M$ and $N$ be subsets of Banach spaces such that there is homeomorphism $h:(M$, weak $) \rightarrow(N$, weak $)$. By applying Corollary 1 to the composition $u$ of the identity map: $(M$, norm $) \rightarrow(M$, weak $)$ and $h$, we see that $M$ is $\sigma$-discrete relative to the norm-topology whenever $N$ is.

A subset of a topological space $X$ is called a Souslin- $\mathscr{F}$ set in $X$ if it is obtained by applying the Souslin operation (i.e., the operation $\mathscr{A}$ ) to closed subsets of $X$ (cf. [3]; such a set is called "analytic" in [2,8]). A metrizable space $M$ is said to be absolute Souslin-F if, whenever a subset $N$ of a metrizable space $X$ is homeomorphic to $M, N$ is a Souslin- $\mathscr{F}$ set in $X$. A Souslin- $\mathscr{F}$ set in a complete metric space is an absolute Souslin- $\mathscr{F}$ space in the relative topology (cf. [8]).

Corollary 3. The property of being an absolute Souslin- $\mathscr{F}$ space of weight $\leq \aleph_{1}$ in the norm topology is a weak-invariant.

Proof. As in the proof of Corollary 2, let $M$ and $N$ be, respectively, subsets of Banach spaces $E$ and $F$, and suppose that there is a homeomorphism $h:(M$, weak $) \rightarrow(N$, weak $)$ and that $M$ is a Souslin- $\mathscr{F}$ set of weight $\leq \aleph_{1}$ in $(E$, norm) . We must show that $(N$, norm) is an absolute Souslin- $\mathscr{F}$ space. Note that $(N$, norm $)$ is of weight $\leq \aleph_{1}$ since the weight relative to the norm topology is a weak-invariant.

The rest of the proof depends heavily on results in [2]. We indicate with asterisks those terms whose definitions are to be found in [2].

We first check that the map $h:(M$, norm $) \rightarrow(N$, norm $)$ is co- $\sigma$-discrete*. Let $\left\{A_{t}: t \in T\right\}$ be a discrete family of subsets of $M$. If $S$ is a selector for the family $\left\{h\left(A_{t}\right): t \in T\right\}$, then $S$ is $\sigma$-discrete by Corollary 2. Since the weight of $(N$, norm $)$ is $\leq \aleph_{1}$, by [5, Remark 2, p. 101], $\left\{h\left(A_{t}\right): t \in T\right\}$ is $\sigma$-discretely decomposable* in $\bigcup\left\{h\left(A_{t}\right): t \in T\right\}$ and hence in $F$ (cf. [2, p. 684]).

The graph $G=\{(x, h(x)): x \in M\}$ is closed in $(M$, norm $) \times(F$, norm $)$, being closed in $(M$, weak $) \times(F$, weak $)$. Since $M \times F$ is a Souslin- $\mathscr{F}$ set in $(E$, norm $) \times(F$, norm $), G$ is a Souslin- $\mathscr{F}$ set in the complete metric space $(E$, norm $) \times(F$, norm $)$. Therefore $(G, \tau)$ is an absolute Souslin- $\mathscr{F}$ space where $\tau$ is the restriction to $G$ of the product of the norm topologies. By [2, Lemma 6.1], the projection (in the second coordinate) $p:(G, \tau) \rightarrow(F$, norm) is co- $\sigma$-discrete*, and therefore, by [2, Corollary 4.2], $p(G)=N$ is an absolute Souslin- $\mathscr{F}$ space in the norm topology. 
Remark. The results of Fleissner [1, Lemma 4.9 and Theorem 5.3] show that, assuming the existence of supercompact cardinals, there is a model of set theory in which the argument used in the proof of Corollary 3 can be repeated without any weight restrictions. Hence under these hypotheses, the property of being an absolute Souslin-F space in the norm topology is a weak-invariant. Is the same true in ZFC?

Example. In Corollary 2, one cannot replace " $\sigma$-discreteness" with "discreteness". To see this, let $K=\mathbb{N} \cup\{\infty\}$, the one-point compactification of $\mathbb{N}$, and let $\mathscr{A}=\{A \subset \mathbb{N}:|A|<\infty\}$. Then $M=\left\{\chi_{A}: A \in \mathscr{A}\right\} \subset C(K)$ is discrete in the norm topology. Let $f: \mathbb{N} \rightarrow R$ be the function given by $f(n)=\frac{1}{n}$ for $n \in \mathbb{N}$, and let $N=\left\{f \chi_{A}: A \in \mathscr{A}\right\} \subset C(K)$. Then ( $M$, weak) and $(N$, weak) are homeomorphic, but each point of $N$ is a limit point of $N$ in the norm topology.

\section{REFERENCES}

1. W. G. Fleissner, An axiom for nonseparable Borel theory, Trans. Amer. Math. Soc. 251 (1979), 309-328.

2. R. W. Hansell, On characterizing non-separable analytic and extended Borel sets as types of continuous images, Proc. London Math. Soc. 28 (1974), 683-699.

3. J. E. Jayne and C. A. Rogers, K-analytic sets, Analytic Sets (Conf., University of Coll., Univ. of London, 1978), Academic Press, London and New York, 1980, pp. 1-181.

4. J. L. Kelley et al., Linear topological spaces, Graduate Texts in Math., vol. 27, SpringerVerlag, New York, Heidelberg, and Berlin, 1975.

5. R. Pol, Note on decompositions of metrizable spaces. I, Fund. Math. 95 (1977), 95-103.

6. A. H. Stone, On $\sigma$-discreteness and Borel isomorphism, Amer. J. Math. 85 (1963), 655-666.

7. M. H. Stone, The generalized Weierstrass approximation theorem, Math. Mag. 21 (1948), 167-184, 237-254.

8. W. Sierpinski, General topology, 2nd ed., Univ. of Toronto Press, Toronto, 1956.

Department of Mathematics, University of Washington, Seattle, Washington 98195 E-mail address, I. Namioka: namioka@math.washingon.edu

Wydzial Mathematyki Uniwersytet Warszawski, Banacha 2, 00-913 Warszawa 59, PolAND 